This is a author version of

D. Schlipf, P. Grau, S. Raach, R. Duraiski, J. Trierweiler, and P. W. Cheng,

"Comparison of Linear and Nonlinear Model Predictive Control of Wind Turbines Using LIDAR,"

published in Proceedings of the American Control Conference, Portland, USA, 2014.

\title{
Comparison of Linear and Nonlinear Model Predictive Control of Wind Turbines Using LIDAR
}

\author{
David Schlipf $^{1}$, Patrick Grau ${ }^{1 \ddagger}$, Steffen Raach ${ }^{1}$, Ricardo Duraiski ${ }^{2}$, Jorge Trierweiler ${ }^{3}$, and Po Wen Cheng ${ }^{1}$
}

\begin{abstract}
Recent developments in remote sensing are offering a promising opportunity to rethink conventional control strategies of wind turbines. With technologies such as LIDAR, the information about the incoming wind field - the main disturbance to the system - can be made available ahead of time. Feedforward control can be easily combined with traditional collective pitch feedback controllers and has been successfully tested on real systems. Nonlinear model predictive controllers adjusting both collective pitch and generator torque can further reduce structural loads in simulations but have higher computational times compared to feedforward or linear model predictive controller. This paper compares a linear and a commercial nonlinear model predictive controller to a baseline controller. On the one hand simulations show that both controller have significant improvements if used along with the preview of the rotor effective wind speed. On the other hand the nonlinear model predictive controller can achieve better results compared to the linear model close to the rated wind speed.
\end{abstract}

\section{INTRODUCTION}

To reduce the structural loads of wind turbines by advanced control is an important issue to lower the cost of energy. Conventional feedback controllers relying on the measured rotational speed can only provide delayed compensation for excitations such as gusts due to the huge inertia of the rotor. This usually results in additional loads on the wind turbine and requires high actuator rates for the disturbance compensation. New remote sensing techniques such as LIDAR (LIght Detection And Ranging) allow a measurement of the wind in front of the wind turbine providing a preview disturbance information, which can be used in new control concepts to improve the disturbance rejection.

Early work on LIDAR-assisted control was reported in [1] and this field of investigation has increased significantly in recent years. While SISO feedforward controllers have been already successfully tested for load reduction on real turbines [2], [3], model predictive controllers are promising to further reduce structural loads, see e.g., [4]-[12]. The present work illustrates on the one hand that wind preview is crucial to achieve significant improvement over a baseline feedback controller. On the other hand a nonlinear model predictive controller (NMPC) exceed the improvements of a

1 Stuttgart Chair of Wind Energy (SWE), Universität Stuttgart, Germany, David.Schlipf/Steffen.Raach/PoWen.Cheng Qifb.uni-stuttgart. de, ${ }^{\ddagger}$ now with Robert Bosch GmbH

${ }^{2}$ TriSolutions - Controle e Otimização de Processos, Porto Alegre, Brazil duraiskietrisolutions.com.br

${ }^{3}$ Universidade Federal do Rio Grande do Sul, Porto Alegre, Brazil jorgedenq.ufrgs.br

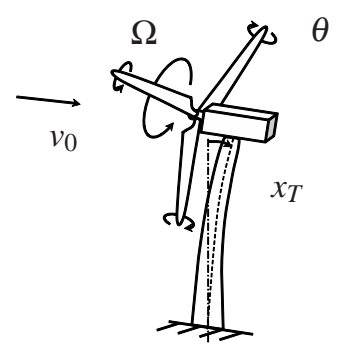

Fig. 1. Degrees of freedom for the reduced nonlinear model.

linear model predictive controller (LMPC) due to the high nonlinearity of the wind turbine close to rated wind speed.

This paper is based on [13] and is organized as follows. Section II and III summarize the modeling of the wind turbine and the LIDAR measurements. Section IV describes the design of an Extended Kalman Filter (EKF), and the different controllers are outlined in Section V. Section VI presents simulation results followed by the conclusions and future work in Section VII.

\section{Modeling of the Wind Turbine}

Classically, aeroelastic codes such as FAST [14] and stochastic wind field generators such as TurbSim [15] are used for simulations in wind energy, providing models close to reality. However, they are too complex to be used for controller design. The internal model for the linear and nonlinear model predictive controller design should be simple enough to allow simulations in a reasonable computation time. At the same time it should be accurate enough to capture the system dynamics that are relevant for wind turbine control. In addition, current remote sensing methods such as LIDAR are not able to measure a detailed three dimensional wind field as used in simulations. But they show promising capabilities to measure the overall rotor effective wind speed [2], [3]. Therefore, modeling details such as the dynamics of individual blades is unnecessary, because these dynamics cannot be predicted if the corresponding disturbance preview is missing. In this section, a reduced nonlinear turbine model with three degrees of freedom and its linearization are presented.

\section{A. Reduced Nonlinear Model}

For the simulations in this work a $5 \mathrm{MW}$ reference wind turbine [16] is reduced to be used as internal model for the controller design. The model has been used in previous studies [9] and is based on [17]. Similar to FAST, it is split 
up into a servo-elastic and an aerodynamic subsystem (see Figure 1).

1) Servo-Elastics: The first tower fore-aft bending mode, the rotational motion, and the collective pitch actuator are modeled linearly as:

$$
\begin{aligned}
J \dot{\Omega}+M_{g} / i_{g b} & =M_{a}\left(\dot{x}_{T}, \Omega, \theta, v_{0}\right) \\
m_{T e} \ddot{x}_{T}+c_{T e} \dot{x}_{T}+k_{T e} x_{T} & =F_{a}\left(\dot{x}_{T}, \Omega, \theta, v_{0}\right) \\
\ddot{\theta}+2 \xi \omega \dot{\theta}+\omega^{2}\left(\theta-\theta_{c}\right) & =0 .
\end{aligned}
$$

The conservation of the angular momentum (1a) gives the equation of motion of the rotor speed $\Omega$. The aerodynamic torque $M_{a}$ accelerates the rotor, depending on the tower top fore-aft displacement $x_{T}$, the effective collective blade pitch angle $\theta$, and the rotor effective wind speed $v_{0}$. The electrical generator torque $M_{g}$ decelerates the rotor, where $i_{g b}$ is the gear box ratio and $J$ is the overall sum of the moments of inertia about the rotation axis. The tower foreaft dynamics are modeled as a mass-spring-damper system (1b), where $F_{a}$ is the aerodynamic thrust and $m_{T e}, c_{T e}$, and $k_{T e}$ are the tower equivalent modal mass, structural damping, and bending stiffness, respectively. Finally, the blade pitch actuator (1c) is a second-order linear model, where $\omega$ is the undamped natural frequency, $\xi$ is the damping factor, and $\theta_{c}$ is the collective blade pitch control input.

2) Aerodynamics: The aerodynamic torque and thrust acting on the rotor with radius $R$ are nonlinear static functions:

$$
\begin{aligned}
M_{a} & =\frac{1}{2} \rho \pi R^{3} \frac{c_{P}(\lambda, \theta)}{\lambda} v_{r e l}^{2} \\
F_{a} & =\frac{1}{2} \rho \pi R^{2} c_{T}(\lambda, \theta) v_{r e l}^{2}
\end{aligned}
$$

where $c_{P}$ and $c_{T}$ are the effective power and thrust coefficients obtained from steady-state simulations and $\rho$ is the air density. The relative wind speed $v_{r e l}$ is defined as a superposition of tower top speed and mean wind speed

$$
v_{r e l}=v_{0}-\dot{x}_{T}
$$

and the tip-speed ratio $\lambda$ is

$$
\lambda=\frac{\Omega R}{v_{r e l}} .
$$

The individual differential equations can be transformed in the state space representation with the system states

$$
x=\left[\begin{array}{lllll}
x_{T} & \dot{x}_{T} & \Omega & \theta & \dot{\theta}
\end{array}\right]^{T},
$$

the control input $u$, and disturbance $d$ :

$$
u=\left[\begin{array}{ll}
M_{g} & \theta_{c}
\end{array}\right]^{T}, d=v_{0}
$$

The system outputs $y$ of the design model are the acceleration of the tower top and the rotor speed accessible from commonly used sensors:

$$
y=\left[\begin{array}{ll}
\ddot{x}_{T} & \Omega
\end{array}\right]^{T} .
$$

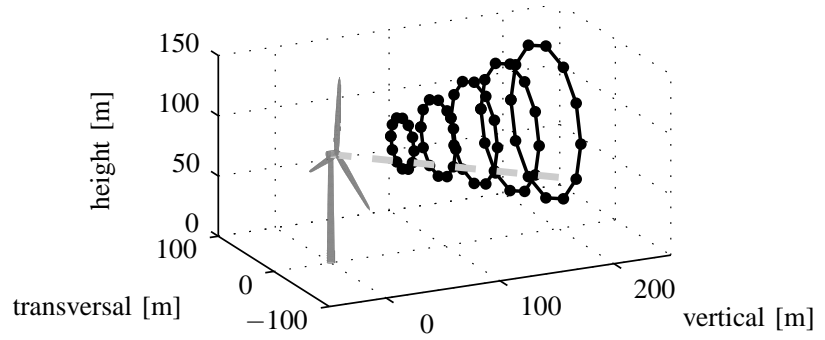

Fig. 2. Used circular scan for the LIDAR measurements.

\section{B. Reduced Linear Model}

In order to design a linear MPC, a linear representation is needed. Therefore, the obtained nonlinear reduced model (2)-(4) is linearized at an equilibrium point defined by $v_{0}$. Then the linear state space representation is

$$
\begin{aligned}
& \Delta \dot{x}=\underbrace{\left[\begin{array}{ccccc}
0 & 1 & 0 & 0 & 0 \\
\frac{-k_{T e}}{m_{T e}} & \frac{-c_{T e}+\partial F_{a} / \partial \dot{x}_{T}}{m_{T g}} & \frac{\partial F_{a} / \partial \Omega}{m_{T \rho}} & \frac{\partial F_{a} / \partial \theta}{m_{T \rho_{j}}} & 0 \\
0 & \frac{\partial M_{a} / \partial \dot{x}_{T}}{J} & \frac{\partial M_{a} / \partial \Omega}{J} & \frac{\partial M_{a} \partial \theta}{J} & 0 \\
0 & 0 & 0 & 0 & 1 \\
0 & 0 & 0 & -\omega^{2} & -2 \xi \omega
\end{array}\right]}_{A} \Delta x \\
& +\underbrace{\left[\begin{array}{cc}
0 & 0 \\
0 & 0 \\
0 & \frac{-1}{i_{g b}} \\
0 & 0 \\
\omega^{2} & 0
\end{array}\right]}_{B_{u}} \Delta u+\underbrace{\left[\begin{array}{c}
0 \\
\frac{\partial F_{a} / \partial v_{0}}{m_{T e}} \\
\frac{\partial M_{a} / \partial v_{0}}{J} \\
0 \\
0
\end{array}\right]}_{B_{d}} \Delta d \\
& \Delta y=\underbrace{\left[\begin{array}{ccccc}
\frac{-k_{T e}}{m_{T e}} & \frac{-c_{T e}+\partial F_{a} / \partial \dot{x}_{T}}{m_{T e}} & \frac{\partial F_{a} / \partial \Omega}{m_{T e}} & \frac{\partial F_{a} / \partial \theta}{m_{T e}} & 0 \\
0 & 0 & 1 & 0 & 0
\end{array}\right]}_{C} \Delta x \\
& +\underbrace{\left[\begin{array}{ll}
0 & 0 \\
0 & 0
\end{array}\right]}_{D_{u}} \Delta u+\underbrace{\left[\begin{array}{c}
\frac{\partial F_{a} / \partial v_{0}}{m_{T e}} \\
0
\end{array}\right]}_{D_{d}} \Delta d
\end{aligned}
$$

The partial derivatives of $F_{a}$ and $M_{a}$ are obtained by fitting polynomials to $c_{P}$ and $c_{T}$ and calculating the corresponding derivatives at the operation point.

\section{Simulated LiDAR MeAsurements}

The preview of the rotor effective wind speed is extracted from the 3D wind fields used for the aero-elastic simulation. Similar to [9] a pulsed system with a circular trajectory is simulated (Figure 2), assuming that the turbulent wind field moves unaffected with the average wind speed and thus is scanned at times corresponding to the 5 distances before it reaches the rotor. Due to the limited points and the limitation to "line-of-sight" measurements, only the low frequencies can be measured correctly. To avoid harmful control action due to uncorrelated frequencies the wind speed preview $v_{0 L}$ is filtered by a low-pass filter designed according to [18]. 


\section{ESTIMATOR DESIGN}

To realize a model based controller all system states have to be available. For a realistic measurement scenario only the rotor speed $\Omega$ and the tower top acceleration $\ddot{x}_{T}$ are assumed to be measurable. An EKF is used to reconstruct the missing states of the nonlinear model considering noise.

It can be separated into the prediction update part and the measurement update part. For the prediction update part the discrete nonlinear system is used

$$
\begin{aligned}
\tilde{x}_{k} & =f\left(\hat{x}_{k-1}, u_{k}\right) \\
\tilde{y}_{k} & =h\left(\tilde{x}_{k}\right),
\end{aligned}
$$

where $\tilde{x}_{k}$ and $\tilde{y}_{k}$ are priori values, $\hat{x}_{k-1}$ is the posteriori estimation from the previous time step and $u_{k}$ the actual input. The measurement update part uses a linearized representation around the actual priori state $\tilde{x}_{k}$. The error equation is

$$
\begin{aligned}
& \tilde{e}_{x_{k}} \approx A \cdot\left(x_{k-1}-\hat{x}_{k-1}\right)+\varepsilon_{k} \\
& \tilde{e}_{y_{k}} \approx H \cdot \tilde{e}_{x_{k}}+\eta_{k},
\end{aligned}
$$

with $A$ and $H$ the Jacobian of the vector fields $f$ and $h$, respectively. $\varepsilon_{k}$ is the covariance matrix $W_{k} Q W_{k}^{T}$ and $\eta_{k}$ $V_{k} R V_{k}^{T}$, where $W_{k}$ is the Jacobian of $f$ with respect to the state noise $w$ and $V_{k}$ is the Jacobian of $h$ with respect to the measurement noise $v$ at time step $k . Q$ and $R$ are variances of the assumed noises. The prediction update part is then

$$
\begin{aligned}
& \hat{x}_{k}^{-}=f\left(\hat{x}_{k-1}, u_{k}\right) \\
& P_{k}^{-}=A_{k} P_{k-1} A_{k}^{T}+W_{k} Q_{k-1} W_{k}^{T},
\end{aligned}
$$

where - means that this value is calculated in the prediction step and will be updated in the measurement update part. Together with the Kalman Gain

$$
K_{k}=P_{k}^{-} H_{k}^{T}\left(H_{k} P_{k}^{-} H_{k}^{T}+V_{k} R V_{k}^{T}\right)^{-1}
$$

the measurement update part is

$$
\begin{aligned}
& \hat{x}_{k}=\hat{x}_{k}^{-}+K_{k}\left(y_{k}-h\left(\hat{x}_{k}^{-}\right)\right) \\
& P_{k}=\left(I-K_{k} H_{k}\right) P_{k}^{-} .
\end{aligned}
$$

Figure 3 depicts a comparison between the estimated and the simulated values.
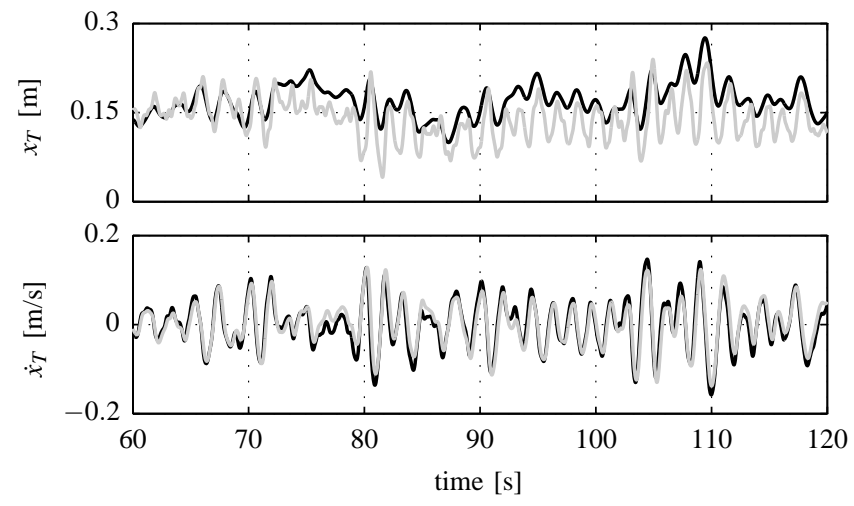

Fig. 3. Comparison between estimated (black) tower fore-aft position and velocity and the simulated values (gray) from a FAST simulation.

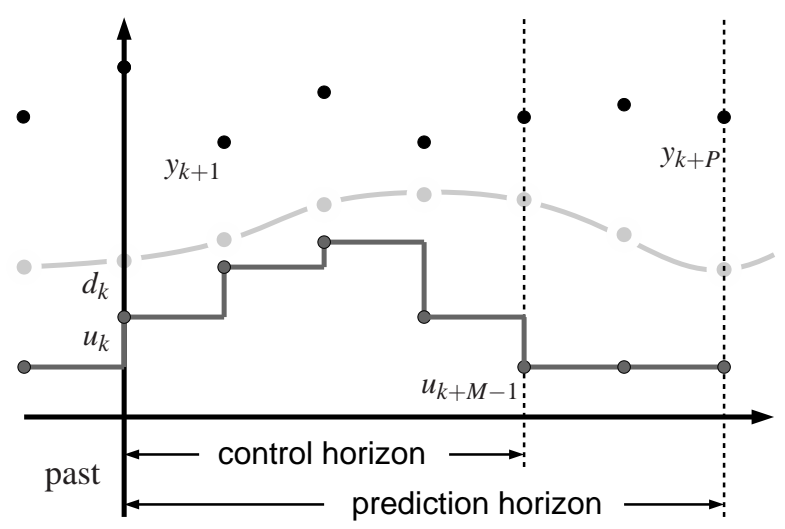

Fig. 4. Principle of the MPC: The projected control inputs $u_{i}$ over a control horizon are optimized, considering the system states $x_{i}$ and the disturbance $d$ over a prediction horizon.

\section{Controller Design}

In this section a Linear (LMPC) and a Nonlinear Model Predictive Controller (NMPC) using the wind speed preview information are derived. Both are solving in principle the same optimal control problem, but using different models and algorithms. The baseline controller (FB) is based on feedback only and is implemented as described in [16], combining a variable speed generator torque controller and a collective pitch controller.

\section{A. The Optimal Control Problem}

The considered optimal wind turbine control problem for the full load region can be described as follows (see Figure 4). The objective is to find the optimal control inputs $u_{i}$ minimizing the cost functional $J$, which is defined as the sum of all deviations from the system outputs $y_{i}$ from the reference values $r$ over the prediction horizon $P$ weighted with $Q$ and the sum of all changes in the control inputs $u_{i}$ over the control horizon $M$ weighted with $R$ :

$$
\begin{array}{ll} 
& \min _{u_{k} \ldots u_{k+M-1}} J \\
\text { with: } & J=\sum_{i=k+1}^{k+P}\left\|Q\left[y_{i}-r\right]\right\|^{2}+\sum_{i=k}^{k+M-1}\left\|R\left[u_{i}-u_{i-1}\right]\right\|^{2}, \\
\text { s.t.: } & \Delta x_{i+1}=A_{i} \Delta x_{i}+B_{u i} \Delta u_{i}+B_{d i} \Delta d_{i} \\
& \Delta y_{i}=C_{i} \Delta x_{i}+D_{u i} \Delta u_{i}+D_{d i} \Delta d_{i} \\
& u_{i}=u_{k+M-1} \quad \forall i=k+M \ldots k+P \\
& u_{\text {min }} \leq u_{i} \leq u_{\max } \quad \forall i=k \ldots k+M-1 \\
& \left|\left(u_{i}-u_{i-1}\right) / \Delta t\right| \leq \dot{u}_{\max } \quad \forall i=k \ldots k+M-1 \\
& y_{\min } \leq y_{i} \leq y_{\max } \quad \forall i=k+1 \ldots k+P .
\end{array}
$$

The control inputs are held constant for the remaining time. The system matrices $A_{i}, B_{u i}, B_{d i}, C_{i}, D_{u i}, D_{d i}$ and deviations $\Delta x_{i}, \Delta y_{i}, \Delta u_{i}, \Delta d_{i}$ are different for the two approaches and will be explained in the next subsections. The reference $r$ and the constraints for the system outputs, control inputs, and for the 
deviations in the control inputs are

$$
\begin{aligned}
& r=\left[0 \frac{\mathrm{m}}{\mathrm{s}^{2}}\right. \\
& u_{\text {min }}=\left[0.9 M_{g, \text { rated }}\right. \\
& u_{\text {max }}=\left[1.1 M_{g, \text { rated }}\right. \\
& \dot{u}_{\text {max }}=\left[15 \frac{\mathrm{kNm}}{\mathrm{s}}\right. \\
& y_{\text {min }}=\left[-0.5 \frac{\mathrm{m}}{\mathrm{s}^{2}}\right. \\
& y_{\max }=\left[0.5 \frac{\mathrm{m}}{\mathrm{s}^{2}}\right. \\
& \left.\Omega_{\text {rated }}\right]^{T} \\
& 0 \mathrm{deg}]^{T} \\
& 90 \mathrm{deg}]^{T} \\
& \left.8 \frac{\mathrm{deg}}{\mathrm{s}}\right]^{T} \\
& \left.0.8 \Omega_{\text {rated }}\right]^{T} \\
& \left.1.2 \Omega_{\text {rated }}\right]^{T},
\end{aligned}
$$

where $\Omega_{\text {rated }}$ and $M_{g, \text { rated }}$ are the rotor speed and generator torque at rated power.

\section{B. Linear Model Predictive Control}

For the LMPC the Model Predictive Control Toolbox [19] and the linear model (5) at the operation point $v_{0}=16 \mathrm{~m} / \mathrm{s}$ was used. In this case, the system matrices $A_{i}, B_{u i}, B_{d i}, C_{i}, D_{u i}, D_{d i}$ in (11) are discretized constant versions of $A, B_{u}, B_{d}, C, D_{u}, D_{d}$ and the deviations $\Delta x_{i}, \Delta y_{i}, \Delta u_{i}, \Delta d_{i}$ denote deviations from the operation point. The system input and outputs were further scaled to speed up the calculation. The used toolbox solves the optimal control problem (11) via Quadratic Programming.

\section{Nonlinear Model Predictive Control}

The algorithm of the Local Linearization on the Trajectory (LLT), shown in [20] and [21], minimizes an objective function by using a nonlinear model, which will be consecutively linearized for each prediction step of the prediction horizon.

The LLT algorithm is oriented at the linear MPC algorithm, in which only one model is used to predict the outputs. The basic idea of the LLT is to linearize a nonlinear model at each point of the prediction horizon and to get $P$ different linearized models. The benefit of this algorithm in comparison to a normal nonlinear MPC-algorithm is that a powerful and fast QP-Solver can be used. The first step is to solve a QP with one linearized model at the actual point to get the first sequence of future inputs $u_{i}$. With this sequence and the nonlinear model the future states $x_{i}$ can be calculated. Then $P$ models are obtained by linearizing along the trajectories. By using the system matrices $A_{i}, B_{u i}, B_{d i}, C_{i}, D_{u i}, D_{d i}$ at the actual linearization point and the deviations from the last

$$
\begin{aligned}
\Delta x_{i} & =x_{i}-x_{i-1}^{L P} & \Delta y_{i} & =y_{i}-y_{i-1}^{L P} \\
\Delta u_{i} & =u_{i}-u_{i-1}^{L P} & \Delta d_{i} & =d_{i}-d_{i-1}^{L P}
\end{aligned}
$$

the QP can be updated and solved again. After that with the new calculated sequence of future inputs, new trajectories of the states can be simulated. This process will be repeated until the changes in $u_{i}$ falls below a certain tolerance. After the control action is applied, the transient response is evaluated and a new trajectory is designed, which is used as a starting trajectory for the next control action determination. Therefore, the actual operational point is used as starting point only in the first control action determination. Every other calculation, after the controller has been started, is performed considering the transient behavior of the process.

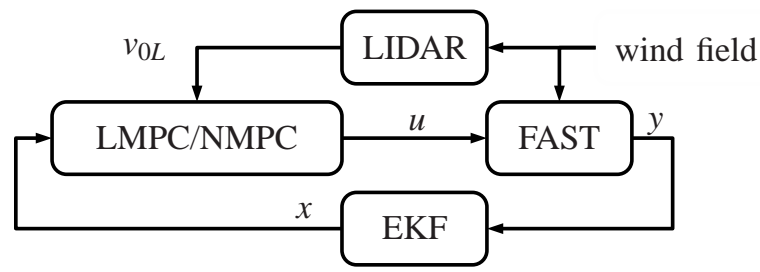

Fig. 5. Overview of the simulation environment.

\section{Simulation Results}

The controllers are compared controlling the FAST $5 \mathrm{MW}$ reference turbine with 16 degrees of freedom and a pitch actuator (1c). The LMPC and the NMPC are simulated with a comparable control and prediction horizon as well as weights for the cost functional. The EKF is used to estimate the tower top position and speed, see Figure 5.

\section{A. Extreme Load Simulations}

In a first evaluation, perfect measurement is assumed and the wind turbine is disturbed by a Extreme Operating Gust (EOG) at $14 \mathrm{~m} / \mathrm{s}$ based on [22]. Here, compared to the proportional-integral (PI) controller both the LMPC and the NMPC are able to compensate the gust and hold the rotor speed almost constant, see Figure 6. Although the aeroelastic model used for the simulation is of higher order compared to the controller design model, the difference in the aerodynamic torque $M_{a}$ in the case of the NMPC is small. For the LMPC, a steady state error can be observed due to the use of an internal model which was linearized at $16 \mathrm{~m} / \mathrm{s}$.

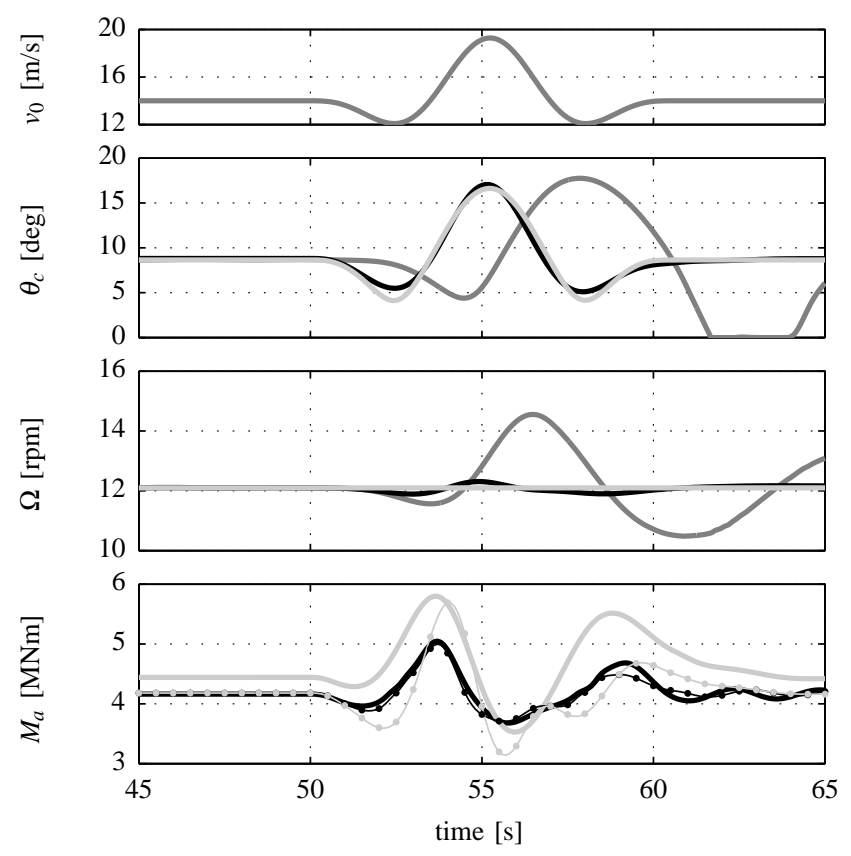

Fig. 6. Top: rotor effective wind speed. Below: pitch angle demand, rotor speed, and aerodynamic torque for PI (dark gray), LMPC (black), and NMPC (light gray). Simulation model (dashed) and internal model (dotted). 

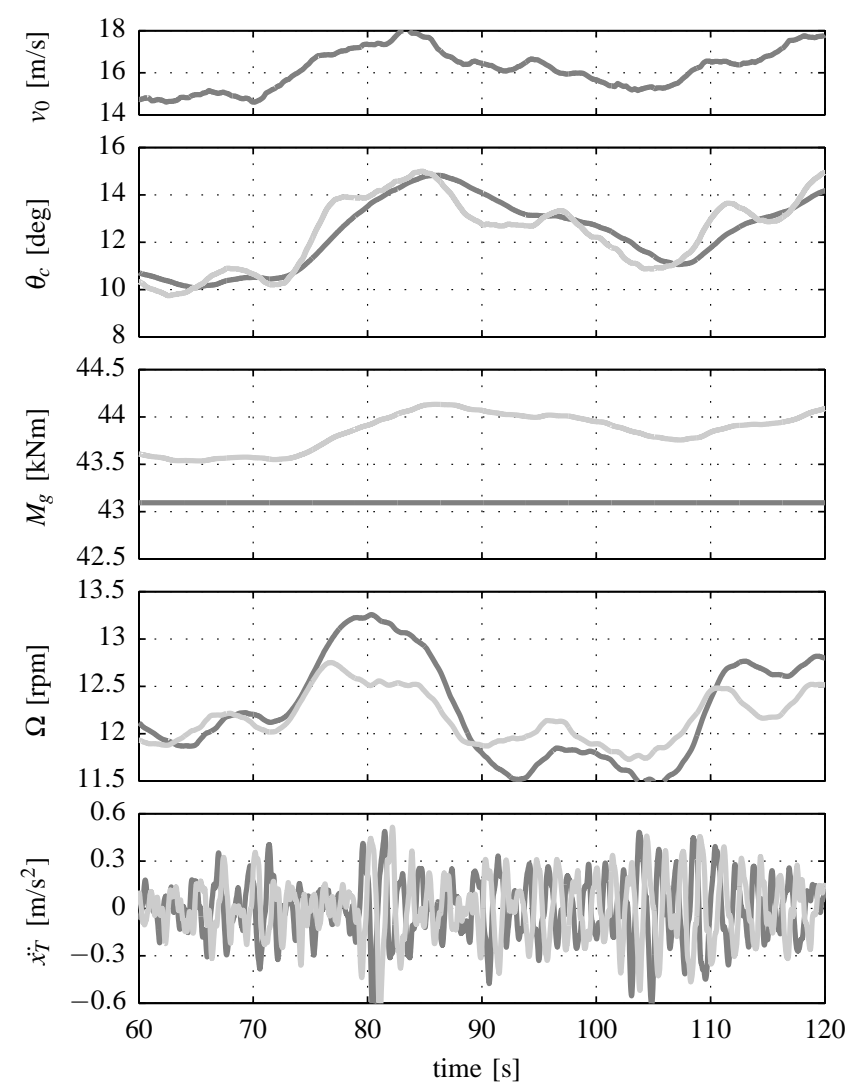

Fig. 7. Top: rotor effective wind speed. Below: pitch angle demand, generator torque, rotor speed, and tower top acceleration for PI (dark gray) and LMPC without wind preview (light gray).

\section{B. Fatigue Load Simulations}

In the next evaluation, a turbulent wind field is used with a mean wind of $16 \mathrm{~m} / \mathrm{s}$ and a turbulent intensity of $18 \%$ for the aero-elastic simulations and simultaneously scaned with the simulated LIDAR system. First, the baseline proportional-integral (PI) controller and the LMPC without wind speed information are compared in Figure 7. The LMPC achieves a slightly better rotor speed regulation using the generator torque to react fast on rotor speed deviations. However, due to the limitation to one linear model, a steady state error in $M_{g}$ occurs. The generator torque for the baseline controller is hold constant for better comparison.

In a next step the baseline PI controller with feed-forward control $(\mathrm{PI}+\mathrm{FF})$ as presented in [23] and tuned for load reduction, the LMPC and NMPC with wind preview are compared, see Figure 8. Because of the advantage of having a forecast all preview controller have an improved rotor speed regulation. The steady state error of the LMPC disappears when used together with the wind speed preview. In Table I can be seen that the NMPC achieves slightly better control performance than feedforward and LMPC controller. Comparing LMPC and NMPC it is remarkable that around the linearized wind speed both achieve nearly similar results. But overall the NMPC achieves better rotor speed regulation especially for low wind speeds where high nonlinearity in the
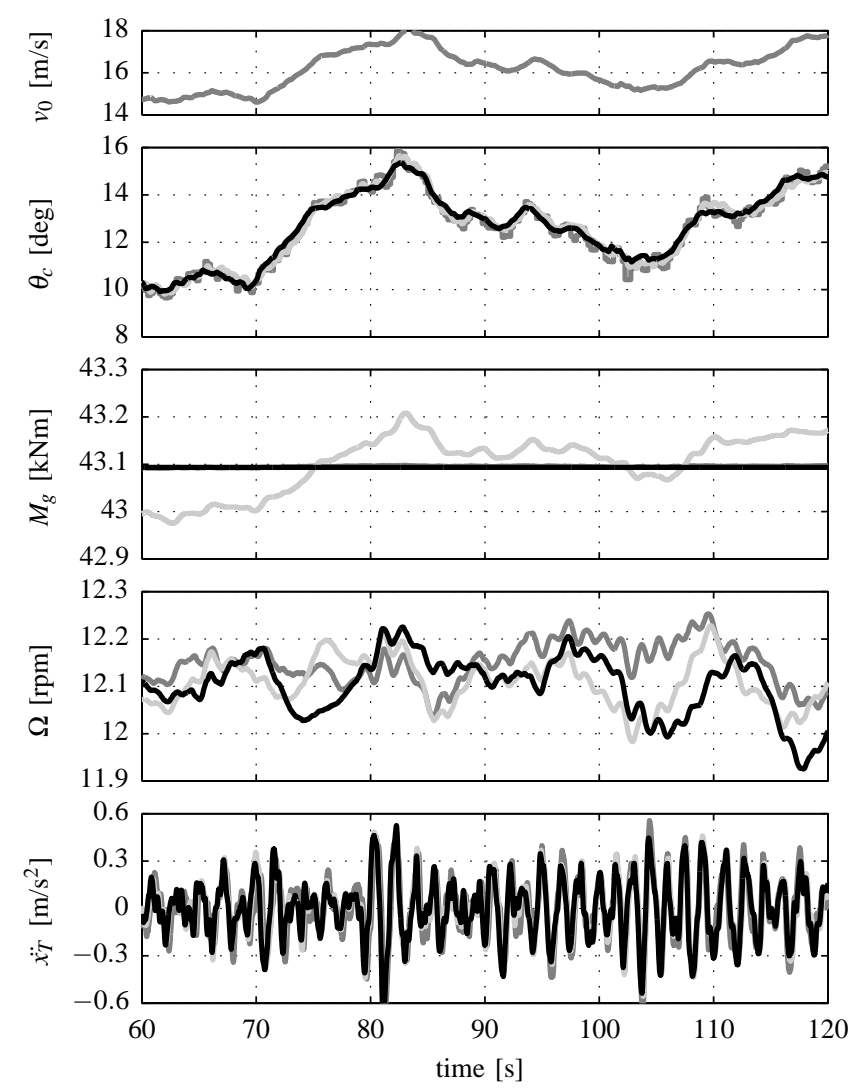

Fig. 8. Top: rotor effective wind speed. Below: pitch angle demand, generator torque, rotor speed, and tower top acceleration for PI (dark gray), LMPC (light gray), and NMPC (black).

aerodynamics occurs. For the NMPC the rotor speed is not always kept to rated rotor speed $\Omega_{\text {rated }}=12.1 \mathrm{rpm}$ because of the trade-off in the cost functional.

In Figure 9, the spectra of the pitch rate, rotor speed and tower top acceleration are depicted. Both MPC controller can reduce the rotor speed spectra below the $1 \mathrm{P}$ (once per revolution) frequency at around $0.2 \mathrm{~Hz}$, but are reaching this performance by a higher control activity. Further investigations have to be made to determine, if frequencies above the $1 \mathrm{P}$ frequency can be omitted by better filtering or estimator tuning, because no benefit is achieved at these frequencies. The spectra of the tower top acceleration can be also be reduced below 1P frequency by both MPC controllers. The reduction is not significant due to the peak in the spectra at the 3P frequency, which is difficult to compensate by a collective pitch control approach.

TABLE I

COMPARISON OF SIMULATION RESULTS.

\begin{tabular}{c||c|c} 
Controller & st. dev. $\Omega[\mathrm{rpm}]$ & st. dev. $\ddot{x}_{T}\left[\mathrm{~m} / \mathrm{s}^{2}\right]$ \\
\hline PI & 0.6193 & 0.1909 \\
LMPC no preview & 0.3226 & 0.1913 \\
PI+FF & 0.0574 & 0.1970 \\
LMPC & 0.0503 & 0.1929 \\
NMPC & 0.0460 & 0.1910
\end{tabular}




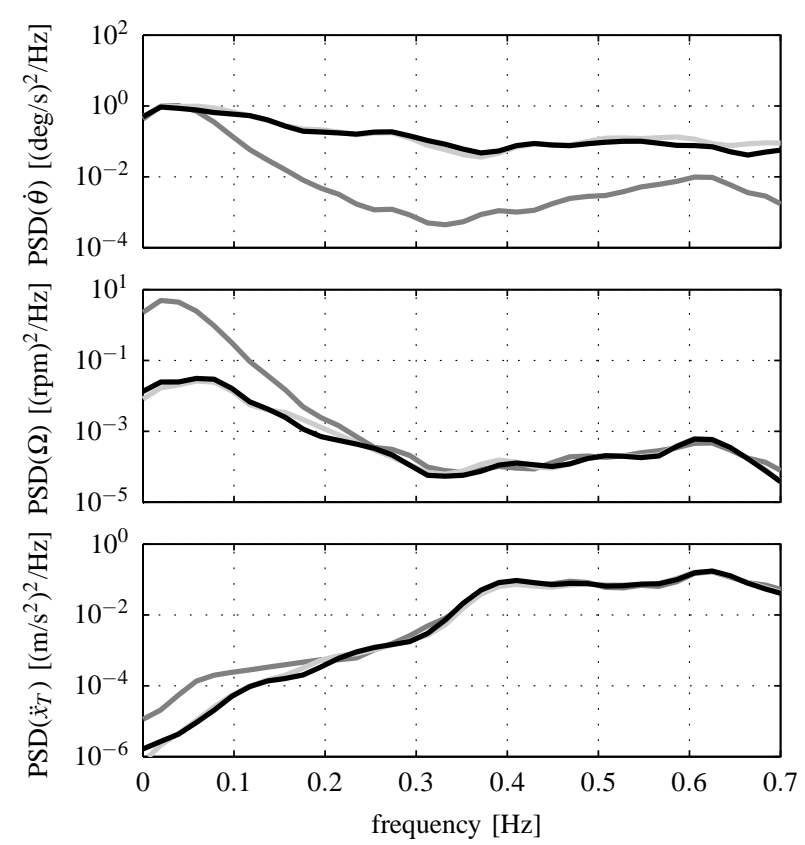

Fig. 9. Spectra of the pitch rate, rotor speed, and tower top acceleration for PI (dark gray) and LMPC (light gray) and NMPC (black).

\section{CONCLUSIONS AND OUTLOOK}

In this paper, we have compared two model predictive controller to a baseline wind turbine controller. Both controller optimize the control trajectories of the collective pitch and the generator torque using simulated LIDAR measurements of the wind inflow. While the linear model predictive controller (LMPC) uses a linear model of one linearization point, the commercial nonlinear model predictive controller (NMPC) uses a nonlinear model, which is linearized for each prediction step. Furthermore, an Extended Kalman Filter is presented, which estimates the tower top displacement and speed, which are usually not available on wind turbines.

Compared to the baseline controller the LMPC is only able to minimize significantly the standard deviation of the rotor speed, if used with wind preview. The NMPC can achieve even better results at wind speeds far from the linearization point of the LMPC, but has higher computational times.

In future work we plan to develop a multivariable feedforward controller for wind speeds around rated wind speed and compare it to the developed MPC. Furthermore, we will investigate the benefit of MPC to floating offshore wind turbines.

\section{ACKNOWLEDGMENT}

Thanks to Matthias Müller, Marcus Reble and Frank Allgöwer from the Institute for Systems Theory and Automatic Control of the Universität Stuttgart for supporting the Diploma thesis which is the basis of the presented work.

Part of this research is funded by the German Federal Ministry for the Environment, Nature Conservation and Nuclear Safety (BMU) in the framework of the joint research project "LIDAR II".

\section{REFERENCES}

[1] M. Harris, M. Hand, and A. Writght, "Lidar for turbine control," Technical Report NREL/TP-500-39154, 2006.

[2] D. Schlipf, P. Fleming, F. Haizmann, A. Scholbrock, M. Hofsäß, A. Wright, and P. W. Cheng, "Field testing of feedforward collective pitch control on the CART2 using a nacelle-based lidar scanner," in Proceedings of The Science of Making Torque from Wind, Oldenburg, Germany, 2012.

[3] A. Scholbrock, P. Fleming, L. Fingersh, A. Wright, D. Schlipf, F. Haizmann, and F. Belen, "Field testing LIDAR based feed-forward controls on the NREL controls advanced research turbine," in 51th AIAA Aerospace Sciences Meeting Including the New Horizons Forum and Aerospace Exposition, Dallas, USA, 2013.

[4] A. A. Kumar and K. A. Stol, "Scheduled model predictive control of a wind turbine," in Proceedings of the 47th AIAA Aerospace Sciences Meeting Including the New Horizons Forum and Aerospace Exposition, Orlando, USA, 2009.

[5] J. Laks, L. Y. Pao, A. Wright, N. Kelley, and B. Jonkman, "Blade pitch control with preview wind measurements," in Proceedings of the 48th AIAA Aerospace Sciences Meeting Including the New Horizons Forum and Aerospace Exposition, Orlando, USA, 2010.

[6] A. Körber and R. King, "Nonlinear model predictive control for wind turbines," in Proceedings of the European Wind Energy Association Annual event, Brussels, Belgium, 2011.

[7] L. C. Henriksen, "Model predictive control of wind turbines," Ph.D. dissertation, Technical University of Denmark, 2011.

[8] D. Schlipf, D. J. Schlipf, and M. Kühn, "Nonlinear model predictive control of wind turbines using LIDAR," Wind Energy, vol. 16, no. 7, pp. 1107-1129, 2013.

[9] D. Schlipf, L. Y. Pao, and P. W. Cheng, "Comparison of feedforward and model predictive control of wind turbines using LIDAR," in Proceedings of the Conference on Decision and Control, Maui, USA, 2012.

[10] J. Aho, L. Pao, and J. Hauser, "Optimal trajectory tracking control for wind turbines during operating region transitions," in Proceedings of the American Control Conference, Washington, USA, 2013.

[11] M. Mirzaei, M. Soltani, N. K. Poulsen, and H. H. Niemann, "An MPC approach to individual pitch control of wind turbines using uncertain lidar measurements," in Proceedings of the European Control Conference, Zürich, Switzerland, 2013.

[12] S. Gros, M. Vukov, and M. Diehl, "A real-time MHE and NMPC scheme for wind turbine control," in Proceedings of the Conference on Decision and Control, Florence, Italy, 2013.

[13] P. Grau, "Model Predictive Control for wind turbines with LIDARtechnology," Master's thesis, Universität Stuttgart, 2011.

[14] J. Jonkman and M. L. Buhl, "FAST user's guide," NREL, Tech. Rep. NREL/EL-500-38230, August 2005.

[15] B. J. Jonkman, "TurbSim user's guide," Technical Report NREL/TP500-46198, September 2009.

[16] J. Jonkman, S. Butterfield, W. Musial, and G. Scott, "Definition of a 5-MW reference wind turbine for offshore system development," Technical Report NREL/TP-500-38060, 2009.

[17] C. L. Bottasso, A. Croce, B. Savini, W. Sirchi, and L. Trainelli, "Aeroservo-elastic modelling and control of wind turbines using finiteelement multibody procedures," Multibody System Dynamics, vol. 16, pp. 291-308, 2006.

[18] D. Schlipf, J. Mann, and P. W. Cheng, "Model of the correlation between lidar systems and wind turbines for lidar assisted control," Journal of Atmospheric and Oceanic Technology, vol. 30, no. 10, pp. 2233-2240, 2013.

[19] A. Bemporad, M. Morari, and L. Ricker, Model Predictive Control Toolbox, The MathWorks, Inc., 2013.

[20] A. R. Secchi, L. G. S. Longhi, and J. O. Trierweiler, "Comparison between linear-adaptive, linear local model network and nonlinear MPC controllers," Latin American Applied Research, vol. 31, pp. 403410, 2001.

[21] R. G. Duraiski, "Controle preditivo não linear utilizando linearizações ao longo da trajetória," Ph.D. dissertation, Universidade Federal do Rio Grande do Sul, Departamento de Engenharia Química, 2001.

[22] IEC 61400-1, Wind turbines - Part 1: Design requirements, International Electrotechnical Commission Std., Rev. third edition, 2005.

[23] D. Schlipf, T. Fischer, C. E. Carcangiu, M. Rossetti, and E. Bossanyi, "Load analysis of look-ahead collective pitch control using LiDAR," in Proceedings of the German Wind Energy Conference DEWEK, Bremen, Germany, 2010. 